\title{
Research Article \\ Heat Conduction Modeling of Fiber Fuse in Single-Mode Optical Fibers
}

\author{
Yoshito Shuto \\ Ofra Project, Iruma 358-0023, Japan \\ Correspondence should be addressed to Yoshito Shuto; ofra@tuba.ocn.ne.jp
}

Received 16 September 2013; Revised 28 December 2013; Accepted 8 January 2014; Published 24 February 2014

Academic Editor: Jorge Diego Marconi

Copyright ( 2014 Yoshito Shuto. This is an open access article distributed under the Creative Commons Attribution License, which permits unrestricted use, distribution, and reproduction in any medium, provided the original work is properly cited.

\begin{abstract}
The unsteady-state thermal conduction process in single-mode optical fiber was studied theoretically with the explicit finitedifference method. We assumed that the vitreous silica optical fiber underwent pyrolysis at elevated temperatures to form $\mathrm{SiO}_{x}$ $(x \sim 1)$. We also proposed a model in which the optical absorption coefficient of the core layer increased with increasing molar concentration of $\mathrm{SiO}_{x}$. The core-center temperature changed suddenly and reached over $3 \times 10^{4} \mathrm{~K}$ when a $1.064-\mu \mathrm{m}$ laser power of $2 \mathrm{~W}$ was input into a short core layer of $40 \mu \mathrm{m}$ length, which was heated at $2923 \mathrm{~K}$. This thermal wave, that is, a fiber fuse, increased in size and propagated toward the light source at a rate of about $0.54 \mathrm{~m} / \mathrm{s}$. The calculated propagation velocity of the fiber fuse was in agreement with the experimental value. Moreover, the average temperature of the radiated region of the core layer gradually approached a temperature of about $5700 \mathrm{~K}$. It was found that the final average temperature was close to the experimentally reported values.
\end{abstract}

\section{Introduction}

By utilizing dense wavelength-division-multiplexed (DWDM) transmission systems using an optical-fiber amplifier, large amount of data can be exchanged at a rate of over $60 \mathrm{Tbit} / \mathrm{s}$ [1]. In connection with this achievement, the danger of the "fiber fuse phenomenon" occurring has been pointed out. This occurs when high-power (W order) optical signals are transmitted in an optical-fiber cable [2].

The fiber fuse phenomenon was first observed in 1987 by British investigators [3-6]. Several review articles [7-9] have been recently published, which cover many aspects of the current understanding of the fiber fuse. Most experimental results of fiber fuse generation have focused on an intensity of $0.35-25 \mathrm{MW} / \mathrm{cm}^{2}$ [3-21]. This is many orders of magnitude below the intrinsic damage limit for silica, which exceeds $10 \mathrm{GW} / \mathrm{cm}^{2}$ [3]. A fiber fuse can be generated by bringing the end of a fiber into contact with an absorbing material or melting a small region of a fiber by using an arc discharge of a fusion splice machine [3]. If a fiber fuse is generated, an intense blue-white flash occurs in the fiber core, and this flash propagates along the core in the direction of the optical power source at a velocity on the order of $1 \mathrm{~m} / \mathrm{s}$. Fuses are terminated by gradually reducing the laser power to provide a termination threshold at which the speed of the fuse is reduced to zero.

When a fiber fuse is generated, the core layer in which the fuse propagates is seriously damaged, and the damaged fiber cannot be used in an optical communication system. The damage is made manifest by periodic or nonperiodic bullet-shaped cavities left in the core [11,20]. It was found that molecular oxygen was released and remained in the cavities while maintaining high pressure (about 4 atmospheres) at room temperature [4].

Dynamics of cavity formation have been investigated since the discovery of the fiber fuse phenomenon. Kashyap reported that the cavity shapes were dependent on the nature of the input laser light (CW or pulses) when the input power was kept at $2 \mathrm{~W}$ average [4]. When CW light was input, the cavities appeared to be oblong and cylindrically symmetric. On the other hand, short asymmetric cavities were formed by entering (mode-locked) pulses with 100 ps FWHM (full width at half maximum), while long bullet-shaped cavities were observed by entering pulses with 190 ps FWHM [4]. Dianov et al. found that the formation of periodic bulletshaped cavities was observed after $20-70 \mu$ s from the passage 
of a plasma leading edge [22,23]. Todoroki reported that fiber fuse propagation was classified into three modes (unstable, unimodal, and cylindrical) according to the plasma volume with respect to the pump beam size [24]. He found that pump power increments and decrements that occurred faster than the plastic flow of the surrounding glass melt led to a cavityfree segment and an irregular cavity pattern, respectively.

These cavities have been considered to be the result of either the classic Rayleigh instability caused by the capillary effect in the molten silica that surrounds a vaporized fiber core [17] or the electrostatic repulsion between negatively charged layers induced at the plasma-molten silica interface [25]. Recently, Todoroki proposed that the bullet-like shape of the cavity train resulted from the formation of an intrinsic bridge inside the hollow silica behind the traveling plasma and the successive compression of detached cavities under a steep temperature gradient along the fiber [8].

It is very important to detect and/or terminate fiber fuse in order to protect expensive equipments for optical communication system. The detection systems for fiber fuse have been recently proposed by two research institutes, which use FBG sensors [26] or an optical time-domain reflectometer [27], respectively. On the other hand, fiber fuse terminators have been investigated by several researchers, which contain tapered fibers $[28,29]$, thermally diffused expanded core (TEC) fibers [30], and a hole-assisted fiber (HAF) [31].

Several hypotheses have been put forward to explain the fiber fuse phenomenon. These include a chemical reaction involving the exothermal formation of germanium (Ge) defects [10], self-propelled self-focusing [3], thermal lensing of the light in the fiber via a solitary thermal shock wave [5], and the radiative collision of $\mathrm{SiO}$ and $\mathrm{O}$ complexes [32].

The similarities between the fiber fuse propagation and the combustion flame propagation were pointed out by Facão et al. [33] and Todoroki [8]. A fast detonation-like mode of fiber fuse propagation with a velocity of $3.2 \mathrm{~km} / \mathrm{s}$ was observed under intense laser radiation intensity of $4000 \mathrm{MW} / \mathrm{cm}^{2}$ [34]. It is noteworthy here that a fiber fuse process itself is not an oxidation process such as the combustion and/or the detonation process. As described in this paper and also pointed out in [7], fiber fuse generation is related to a reduction (pyrolysis) process of $\mathrm{SiO}_{2}$.

The optical absorption coefficient $\alpha$ of a fiber core at high temperatures is closely related to the generation of the fiber fuse. Kashyap reported a remarkable increase in the $\alpha$ value of a Ge-doped silica core above the critical temperature $T_{0}(\sim 1323 \mathrm{~K})$, while the $\alpha$ value of about $0 \mathrm{~dB} / \mathrm{km}$ at room temperature remained unchanged until the temperature $(T)$ approached $T_{0}$ [4]. The $\alpha$ value increased by nearly $1900 \mathrm{~dB} / \mathrm{km}\left(\sim 0.44 \mathrm{~m}^{-1}\right)$ at a wavelength of $1.064 \mu \mathrm{m}$ when $T$ changed from $T_{0}$ to $T_{0}+50 \mathrm{~K}$. Furthermore, Kashyap et al. reported that the best fit between the experimental and theoretical fiber fuse velocities was obtained when the $\alpha$ value of the Ge-doped silica core at high temperatures of above $T_{0}$ was fixed to be $4.0 \times 10^{4} \mathrm{~m}^{-1}$ at $1.064 \mu \mathrm{m}$ [13].

Hand and Russell found that this phenomenon was initiated by the generation of large numbers of Ge-related defects at high temperatures of above $1273 \mathrm{~K}$, and the $\alpha$ values at a wavelength of $0.5 \mu \mathrm{m}$ obtained at temperatures of below $1873 \mathrm{~K}$ were modeled quite accurately using an Arrhenius equation $[5,6]$. By contrast, they reported that the best fit between the experimental and theoretical fiber fuse velocities was obtained when the $\alpha$ value of the Ge-doped silica core at $2293 \mathrm{~K}$ was assumed to be $5.6 \times 10^{4} \mathrm{~m}^{-1}$ at $0.5 \mu \mathrm{m}$ [5]. This large $\alpha$ value, however, could not be estimated using their Arrhenius equation [6]. As the focal length $F$ of thermal lens effect is inversely as the $\alpha$ value [35], large $\alpha$ value of $5.6 \times$ $10^{4} \mathrm{~m}^{-1}$ is necessary to obtain small $F$ value of $10 \mu \mathrm{m}$ order, which is comparable with observed interval and/or large front size of the cavities (see the Appendix).

Furthermore, Hand and Russell reported that the electrical conductivity $\sigma$ of the fiber core increased with the temperature and that the hot spot at the fiber fuse center was plasma-like [5]. Kashyap considered that the large $\alpha$ values may be attributable to an increase in the $\sigma$ value of the fiber core at high temperatures of above $T_{0}[4]$.

It is well known that silica glass is a good insulator at room temperature, and the electrical conductivity in silica glass below $1073 \mathrm{~K}$ is due to positively charged alkali ions moving under the influence of an applied field [36, 37]. The ionic conduction in the glass is not related solely to optical absorption.

We previously investigated the optical absorption mechanism causing the increase in the $\sigma$ value and reported the relationship between $\sigma$ and $\alpha$ in silica glass at high temperatures of above $1273 \mathrm{~K}$ [18]. It was found that the increase in loss observed at $1.064 \mu \mathrm{m}$ can be well explained by the electronic conductivity induced by the thermal ionization of a Ge-doped silica core, and it is not directly related to the absorption of $\mathrm{Ge} E^{\prime}$ centers. However, the calculated $\alpha$ values resulting from the electronic conductivity at $1.064 \mu \mathrm{m}$ were of $10^{2} \mathrm{~m}^{-1}$ order at $2873 \mathrm{~K}$, about two orders smaller than the $\alpha$ values $\left(1.0-4.0 \times 10^{4} \mathrm{~m}^{-1}\right)$ reported by Kashyap et al. $[4,13]$. Therefore, we need another mechanism to explain the increase in loss at high temperatures of above $2273 \mathrm{~K}$ to account for the large $\left(10^{4} \mathrm{~m}^{-1}\right.$ order $) \alpha$ values.

To satisfy this requirement, we proposed a thermochemical $\mathrm{SiO}$ production model in 2004 [18]. Using this model, we theoretically estimated large $\alpha$ values of $10^{4} \mathrm{~m}^{-1}$ order as a result of $\mathrm{SiO}$ absorption at high temperatures of $2800 \mathrm{~K}$ or above. This model was able to quantitatively explain the relation between the fiber fuse propagation velocity and the incident laser power intensity previously reported by other research institutions.

On the other hand, since the parameters (particularly the light-absorbing parameter) used for the numerical simulation were not optimized, the shortcoming that the maximum temperature of the core center obtained by calculation became unusually high $\left(10^{5} \mathrm{~K}\right.$ order $)$ was observed. We have improved this model by optimizing several parameters required for the numerical computation.

In this paper, we describe the improved model in detail. That is, we explain the mechanism of the increased absorption in optical fibers at high temperatures due to the thermochemical production of $\mathrm{SiO}_{x}$ and estimate high-temperature $\alpha$ values at a laser wavelength of $1.064 \mu \mathrm{m}$. Then, using these 
values, we theoretically study the nonsteady-state thermal conduction process in a single-mode optical fiber using the explicit finite-difference technique.

\section{High-Temperature Optical Absorption in Optical Fibers}

2.1. Effect of $\mathrm{SiO}_{x}$ Formation on Absorption. It has been reported that, at elevated temperatures, silica glass is thermally decomposed by the reaction [38]

$$
\mathrm{SiO}_{2} \rightleftarrows \mathrm{SiO}_{x}+\left(\frac{x}{2}\right) \mathrm{O}_{2} .
$$

Among the reductants of the silica generated by this pyrolysis reaction with the formula $\mathrm{SiO}_{x}$, the most thermally stable material is $\mathrm{SiO}(x=1)$.

The internal core space heated at the elevated temperature is shielded from the external conditions. Thus, with increasing temperature, the internal pressure increases and the internal volume decreases. Dianov et al. reported that the internal core temperature is about $10000 \mathrm{~K}$ and the internal pressure is about 10000 atmospheres at the time of fiber fuse evolution [29]. It is thought that $\mathrm{SiO}_{x}$ generated under the high-temperature and high-pressure conditions is densely packed into the internal core space because it is not allowed to expand, and it exists in a liquid-like form. For this reason, it is thought that the optical absorption spectrum of the high density $\mathrm{SiO}_{x}$ in the core space is similar to that of solid $\mathrm{SiO}_{x}$.

Philipp reported that the optical absorption spectrum of a $\mathrm{SiO}_{x}$ film is similar to that of amorphous $\mathrm{Si}$ because of the many $\mathrm{Si}-\mathrm{Si}$ bonds in a $\mathrm{SiO}_{x}$ film and that the absorption coefficient $\alpha_{\mathrm{SiO}}$ for $\mathrm{SiO}(x=1)$ near the threshold energy should be about one-twentieth of that for amorphous $\operatorname{Si} \alpha_{\mathrm{Si}}$ [39]. Furthermore, Philipp estimated the theoretical concentration $f_{\mathrm{Si}}$ of $\mathrm{Si}-\left(\mathrm{Si}_{4}\right)$ when the five possible tetrahedral conformations centering on $\mathrm{Si}, \mathrm{Si}-\left(\mathrm{Si}_{4}\right), \mathrm{Si}-\left(\mathrm{Si}_{3} \mathrm{O}\right), \mathrm{Si}-$ $\left(\mathrm{Si}_{2} \mathrm{O}_{2}\right), \mathrm{Si}-\left(\mathrm{SiO}_{3}\right)$, and $\mathrm{Si}-\left(\mathrm{O}_{4}\right)$, were completely distributed at random within amorphous $\mathrm{SiO}_{x}$ [40]. The relationship between $f_{\mathrm{Si}}$ and $x$ for $\mathrm{SiO}_{x}$ is illustrated in Figure 1. According to Figure $1, f_{\mathrm{Si}}$ for a $\mathrm{SiO}(x=1)$ film is $6.25 \%(=1 / 16)$. This is very close to the factor by which the $\alpha$ value was reduced (about 1/20) as reported by Philipp [39].

The optical absorption near the absorption edge of a $\mathrm{SiO}_{x}$ film is dominated by that of amorphous $\mathrm{Si}$ with the $\mathrm{Si}-\left(\mathrm{Si}_{4}\right)$ tetrahedral conformation, and the optical absorption coefficient $\alpha_{\mathrm{SiO}_{x}}$ near the absorption edge of a $\mathrm{SiO}_{x}$ film can be calculated by multiplying the value of $\alpha_{\mathrm{Si}}$ for amorphous Si by $f_{\mathrm{Si}}$. That is, if the production rate of $\mathrm{SiO}_{x}$ given by (1) is denoted by $g_{\mathrm{SiO}_{x}}$, then $\alpha_{\mathrm{SiO}_{x}}$ at temperature $T$ is given by

$$
\alpha_{\mathrm{SiO}_{x}}(T)=g_{\mathrm{SiO}_{x}}(T) f_{\mathrm{Si}}(x) \alpha_{\mathrm{Si}}(T) .
$$

2.2. Production Rate of $\mathrm{SiO}_{x}$. The most thermally stable compound in $\mathrm{SiO}_{x}$ generated via (1) is $\mathrm{SiO}(x=1)$. Therefore, the production rate $g_{\mathrm{SiO}_{x}}$ of $\mathrm{SiO}_{x}$ in (2) is assumed to be almost equal to the production rate $g_{\mathrm{SiO}}$ of $\mathrm{SiO}$.

$g_{\mathrm{SiO}}$ at temperature $T$ can be calculated as the ratio of the molar concentration $c_{\mathrm{SiO}}$ of $\mathrm{SiO}$ at temperature $T$ to the



FIgURE 1: Relative content of $\mathrm{Si}-\left(\mathrm{Si}_{4}\right)$ in $\mathrm{SiO}_{x}$.

maximum value $c_{\mathrm{SiO}}^{0}$. This $c_{\mathrm{SiO}}^{0}$ is the molar concentration when $\mathrm{SiO}_{2}$ changes to $\mathrm{SiO}$ via (1) with a yield of about $100 \%$.

We assumed that the pyrolysis reaction system given by (1) reaches its equilibrium state during fiber fuse propagation. The equilibrium constant for (1) is denoted as $K_{c}$. The value of $c_{\mathrm{SiO}}$ at temperature $T$ was calculated using $K_{c}$ as described below.

First, the initial molar concentration $c_{\mathrm{SiO}_{2}}^{s}$ $\left(=0.0366 \mathrm{~mol} \mathrm{~cm}^{-3}\right)$ is denoted by $a$. We consider the case that the $\mathrm{SiO}_{2}$ concentration decreases to $a-y$ via the pyrolysis reaction of (1). In this case, the molar concentration of $\mathrm{SiO}$ and the molar concentration $c_{\mathrm{O}_{2}}$ of $\mathrm{O}_{2}$ are expressed as $y$ and $y / 2$, respectively. $K_{c}$ is given in terms of $a$ and $y$ as follows:

$$
K_{c}=\frac{c_{\mathrm{SiO}} c_{\mathrm{O}_{2}}^{1 / 2}}{c_{\mathrm{SiO}_{2}}}=\frac{y(y / 2)^{1 / 2}}{a-y} .
$$

Rearranging (3), we obtain a cubic equation in $y$. The solution of this equation is given by

$$
y=\sqrt[3]{C}+\frac{4 K_{c}^{2}}{3 \sqrt[3]{C}}\left(\frac{K_{c}^{2}}{3}-a\right)+\frac{2 K_{c}^{2}}{3},
$$

where

$$
\begin{aligned}
C= & a^{2} K_{c}^{2}-\frac{4}{3} a K_{c}^{4}+\frac{8}{27} K_{c}^{6} \\
& +a^{2} K_{c}^{2} \sqrt{1-\frac{8 K_{c}^{2}}{27 a}} .
\end{aligned}
$$

It is well known that the equilibrium constant $K_{c}$ is related to the standard Gibbs energy change $\Delta_{r} G^{0}$ for (1). The relationship between $K_{c}$ and $\Delta_{r} G^{0}$ is given by [41]

$$
\ln K_{c}=\frac{-\Delta_{r} G^{0}}{R T},
$$




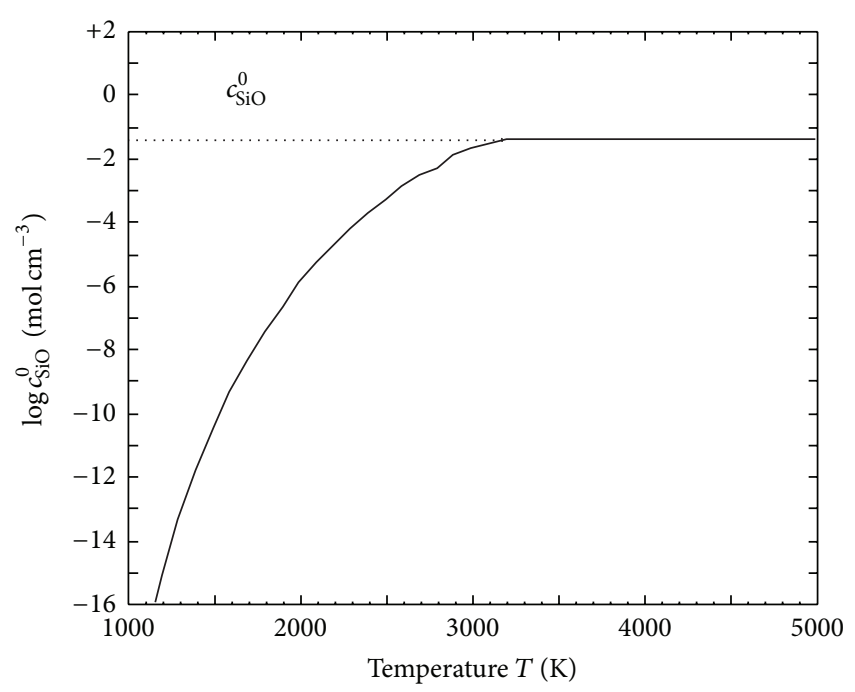

Figure 2: Molar concentration of $\mathrm{SiO}$ versus temperature.

where $R$ is the gas constant. The $\Delta_{r} G^{0}$ value for (1) is given by

$$
\begin{aligned}
\Delta_{r} G^{0}= & \Delta_{f} G_{\mathrm{SiO}}^{0}+\left(\frac{1}{2}\right) \Delta_{f} G_{\mathrm{O}_{2}}^{0} \\
& -\Delta_{f} G_{\mathrm{SiO}_{2}}^{0},
\end{aligned}
$$

where $\Delta_{f} G^{0}$ is the standard production Gibbs energy of a reactant and/or a product.

Vitreous silica $\left(\mathrm{SiO}_{2}\right)$ is a solid at the standard temperature $(298.15 \mathrm{~K})$. It melts at high temperatures of above $1996 \mathrm{~K}$ and becomes a liquid. It also becomes a vapor at temperatures of above $3000 \mathrm{~K}$.

The standard production Gibbs energies $\Delta_{f} G_{\mathrm{SiO}_{2}}^{0}, \Delta_{f} G_{\mathrm{SiO}}^{0}$, and $\Delta_{f} G_{\mathrm{O}_{2}}^{0}$ in each phase have been published [42]. Thus, using these $\Delta_{f} G^{0}$ values, we first calculated the standard Gibbs energy change $\Delta_{r} G^{0}$ for (1). Next, we calculated $K_{c}$ by substituting the $\Delta_{r} G^{0}$ value into (6). In this way, we computed $c_{\mathrm{SiO}}(=y)$ at temperature $T$ by substituting $K_{c}$ and $a\left(=0.0366 \mathrm{~mol} \mathrm{~cm}^{-3}\right)$ into (4) and (5). The relationship between $c_{\mathrm{SiO}}$ and $T$ is shown in Figure 2. $c_{\mathrm{SiO}}$ increases with increasing $T$ and gradually approaches its maximum value $\left(c_{\mathrm{SiO}}^{0} \cong 0.0366 \mathrm{~mol} \mathrm{~cm}^{-3}\right)$ at $T$ of about $3200 \mathrm{~K}$.

Therefore, $g_{\mathrm{SiO}_{x}}$ at temperature $T$ was estimated by dividing the value of $c_{\mathrm{SiO}}$ calculated above by $c_{\mathrm{SiO}}^{0}$ $\left(\cong 0.0366 \mathrm{~mol} \mathrm{~cm}^{-3}\right)$.

2.3. Absorption Coefficient of Amorphous Si. The optical absorption spectrum of amorphous Si was reported by Brodsky et al. [43]. The absorption coefficient $\alpha_{\mathrm{Si}}$ of amorphous $\mathrm{Si}$ in (2) was estimated as follows.

First, we consider the absorption coefficient $\alpha_{\mathrm{Si}}$ of an interband transition region, where the photon energy $\hbar \omega$ is larger than the energy gap $E_{g}(=1.26 \mathrm{eV})$ of amorphous Si. In this case, the values of $\alpha_{\mathrm{Si}}\left(\mathrm{cm}^{-1}\right.$ unit) near $E_{g}$ are given by

$$
\alpha_{\mathrm{Si}}(\omega)=B \frac{\left(\hbar \omega-E_{g}\right)^{2}}{\hbar \omega},
$$

where the parameter $B$ is $5.06 \times 10^{5} \mathrm{~cm}^{-1} \mathrm{eV}^{-1}$. The $\alpha_{\mathrm{Si}}$ values calculated by (8) are in good agreement with the experimental values reported by Brodsky et al. [43] for the case of $\hbar \omega>$ $1.5 \mathrm{eV}$.

Next, we consider the values of $\alpha_{\mathrm{Si}}$ in the low-energy region, where $\hbar \omega<E_{g}$. In this region, an absorption edge spectrum is broadened as a result of the interaction between optical phonons and electrons (or excitons). $\alpha_{\mathrm{Si}}$ in this region exhibits exponential behavior (referred to as an "exponential tail" or "Urbach tail") as follows [44]:

$$
\alpha_{\mathrm{Si}}(\omega)=\alpha_{0} \exp \left[\frac{\gamma\left(\hbar \omega-E_{g}\right)}{k T^{*}}\right],
$$

where $\alpha_{0}$ and $\gamma$ are parameters and $k$ is the Boltzmann constant. $T^{*}$ is the effective temperature. If the characteristic temperature of phonons is denoted by $\theta$, then $T^{*}$ is given by [45]

$$
T^{*}=\frac{\theta}{2} \operatorname{coth}\left(\frac{\theta}{2 T}\right) .
$$

For $\mathrm{Si}, \theta=600 \mathrm{~K}$ [46]. $\alpha_{0}$ and $\gamma$ are estimated to be $9.016 \times 10^{3} \mathrm{~cm}^{-1}$ and 0.14 , respectively, by simulation using the experimental $\alpha_{\mathrm{Si}}$ values reported by Brodsky et al. [43].

It is known that the energy gap $E_{g}$ of Si decreases linearly with increasing $T$ at $T>200 \mathrm{~K}$ [47]. The temperature dependence of $E_{g}$ (eV unit) at $T>300 \mathrm{~K}$ is given by

$$
E_{g}=1.26-\beta T,
$$

where $\beta$ is the temperature coefficient of the energy gap and takes a value of $4.2 \times 10^{-4} \mathrm{eV} / \mathrm{K}$ [48].

2.4. Absorption Coefficient of $\mathrm{SiO}_{x}$. On the basis of the above results, the temperature dependence of $\alpha_{\mathrm{SiO}_{x}}$ at $1.064 \mu \mathrm{m}$ ( $\hbar \omega=1.17 \mathrm{eV}$ ) was calculated using (2) and the $c_{\mathrm{SiO}}$ values shown in Figure 2. $f_{\mathrm{Si}}=0.0625(x=1)$ was used in the calculation. The results are shown in Figure 3.

The $\alpha_{\mathrm{SiO}_{x}}$ values (shown as the thick solid line in Figure 3) are about $1.5 \times 10^{4} \mathrm{~m}^{-1}$ at $2800 \mathrm{~K}$ and about $3.5 \times 10^{4} \mathrm{~m}^{-1}$ at $2950 \mathrm{~K}$. These values are close to $4 \times 10^{4} \mathrm{~m}^{-1}$ (shown as the dotted line in Figure 3), which was estimated by simulation using the finite element method [13].

Moreover, it turns out that $\alpha_{\mathrm{SiO}_{x}}$ reaches its maximum value (about $9.47 \times 10^{4} \mathrm{~m}^{-1}$ ) at about $3150 \mathrm{~K}$ then decreases gradually with increasing temperature.

Compared with the stepwise shape (dotted line in Figure 3 ) of the absorption coefficient assumed by Kashyap et al., the maximum value (about $9.47 \times 10^{4} \mathrm{~m}^{-1}$ ) of $\alpha_{\mathrm{SiO}_{x}}$ is about 2.4 times the assumed value $\left(4 \times 10^{4} \mathrm{~m}^{-1}\right)$, and the graph has the shape of a somewhat distorted echelon form. 




FIgURE 3: Absorption coefficient of $\mathrm{SiO}_{x}$ at $1.064 \mu \mathrm{m}$ versus temperature. The thick solid line was calculated using (2). The dotted line shows the values estimated by Kashyap et al.



Figure 4: Hot zone in the core layer.

As mentioned above, $\mathrm{SiO}_{x}$ is produced in the pyrolysis of vitreous silica, and it induces a very large amount of optical absorption with a large absorption coefficient of $10^{4} \mathrm{~m}^{-1}$ order at high temperatures of above $2800 \mathrm{~K}$. It is thought that the large amount of optical absorption at high temperatures is the cause of the genesis of the fiber fuse phenomenon.

We investigated the thermal conduction behavior within a single-mode (SM) optical fiber by numerical computation using the thermochemical $\mathrm{SiO}_{x}$ production model. In the next section, we describe the results of some numerical calculations related to the thermal conduction process in an SM optical fiber.

\section{Simulation of Fiber Fuse in SM Optical Fiber}

3.1. Heat Conduction in SM Optical Fiber. We assume the SM optical fiber to have a radius of $r_{f}$ and to be in an atmosphere with temperature $T=T_{a}$. We also assume that part of the core layer of length $\Delta L$ is heated to a temperature of $T_{c}^{0}\left(>T_{a}\right)$ (see Figure 4). Such a region, called the "hot zone" in Figure 4, can be generated by heating the fiber end faces using the arc discharge of a fusion splice machine.

As explained above, the optical absorption coefficient $\alpha$ of the core layer in an optical fiber is a function of temperature $T$, and $\alpha$ increases with increasing $T$. In the hot zone in Figure 4, the $\alpha$ values are larger than those in the remainder of the core region because of its elevated temperature. Thus, as light propagates along the positive direction (away from the light source) in this zone, considerable heat is produced by light absorption.

In the case of a heat source in part of the core layer, the nonsteady heat conduction equation for the temperature field $T(r, z, t)$ in the SM optical fiber is given by [49]

$$
\rho C_{p} \frac{\partial T}{\partial t}=\lambda\left(\frac{\partial^{2} T}{\partial r^{2}}+\frac{1}{r} \frac{\partial T}{\partial r}+\frac{\partial^{2} T}{\partial z^{2}}\right)+\dot{Q}
$$

where $\rho, C_{p}$, and $\lambda$ are the density, specific heat, and thermal conductivity of the fiber, respectively. The values of $\rho, C_{p}$, and $\lambda$ used for the calculation are described in the next subsection.

The last term $\dot{Q}$ in (12) represents the heat source resulting from light absorption, which is only required for the hot zone in the fiber core. $\dot{Q}$ can be expressed by

$$
\dot{Q}=\alpha I,
$$

where $I$ is the optical power intensity in the core layer, which can be estimated by dividing the incident optical power $P$ by the effective area $A_{\text {eff }}$ of the fiber.

3.2. Heat Conduction Parameters. In the heat conduction calculation for SM optical fibers, we used the following values of $\lambda\left(\mathrm{W} \mathrm{m}^{-1} \mathrm{~K}^{-1}\right), \rho\left(\mathrm{kg} \mathrm{m}^{-3}\right)$, and $C_{p}\left(\mathrm{~J} \mathrm{~kg}^{-1} \mathrm{~K}^{-1}\right)$ in each temperature range. The unit of $T$ is $\mathrm{K}$ (Kelvin).

(1) Parameters in the temperature range from room temperature ( $298 \mathrm{~K})$ to $1996 \mathrm{~K}$ [50] are as follows:

$$
\begin{gathered}
C_{p}=1194.564+31.541 \times 10^{-3} \mathrm{~T} \\
-651.396 \times 10^{5} \mathrm{~T}^{-2}, \\
\lambda=9.2 \\
\rho=2200.0 .
\end{gathered}
$$

(2) Parameters in the temperature range from 1996 to $3000 \mathrm{~K}[50]$ are as follows:

$$
\begin{gathered}
C_{p}=1430.490, \\
\lambda=9.2, \\
\rho=2200.0 .
\end{gathered}
$$

(3) Parameters for $T>3000 \mathrm{~K}$ are as follows:

$$
\begin{gathered}
C_{p}=844.4, \\
\lambda=0.0025247 \sqrt{T}+1.84275 \times 10^{-13} T^{5 / 2}, \\
\rho=2024.0 .
\end{gathered}
$$

In (3), the first term in the expression for $\lambda$ was estimated by substituting the parameters for $\mathrm{SiO}$ in the following equation, which represents the thermal conductivity of diatomic molecules derived from the kinetic theory of gases [51]:

$$
\lambda=\frac{5}{3 \sigma^{2}} \sqrt{\left(\frac{k}{\pi}\right)^{3} \frac{N_{A}}{M}} \sqrt{T},
$$


where $N_{A}$ is Avogadro's number, $M(=44.0854)$ is the molecular weight of $\mathrm{SiO}$, and $\sigma(=1.5 \AA)$ is half of the collision diameter.

Moreover, in (3), the second term in the expression for $\lambda$ was estimated by multiplying the following thermal conductivity equation for weakly ionized gas plasma [52] by the correction factor of $1 / 20$ :

$$
\lambda=\frac{5 N_{e} k^{2} T}{2 m_{e} \nu_{c}},
$$

where $m_{e}$ is the electron mass, $N_{e}$ is the number density of electrons in the plasma, and $v_{c}$ is the collision frequency. If we assume that electrons mainly collide with ions with a charge of +1 in the plasma, then $\nu_{c}$ is given by [53]

$$
\nu_{c}=\sqrt{\frac{2}{9 \pi}} \frac{N_{e} e^{4}}{\varepsilon_{0}^{2} m_{f e}^{2}}\left(\frac{m_{e}}{3 k T}\right)^{3 / 2} \ln \Lambda,
$$

where $e$ is the electron charge and $\varepsilon_{0}$ is the permittivity of free space. $\ln \Lambda$ is the so-called Coulomb logarithm, and it takes values of 4-34 in the electron temperature range of $10^{2}-10^{8} \mathrm{~K}$ and the $N_{e}$ range of $1-10^{24} \mathrm{~cm}^{-3}$ [54]. In our calculation, we used $\ln \Lambda=16.155$, which corresponds to the case of $T \sim$ $10^{4} \mathrm{~K}$ and $N_{e} \sim 10^{6} \mathrm{~cm}^{-3}$.

3.3. Boundary and Initial Conditions for Heat Conduction. We solved (12) using the explicit finite-difference method (FDM) [55] under the boundary and initial conditions described below.

The area in the numerical calculation had a length of $2 L(=4 \mathrm{~cm})$ in the axial $(z)$ direction and a width of $2 r_{f}$ $(=125 \mu \mathrm{m})$ in the radial $(r)$ direction. There were 24 and 2000 divisions in the $r$ and $z$ directions, respectively, and we set the calculation time interval to $1 \mu \mathrm{s}$. We assumed that the hot zone was located at the center of the fiber (length $2 L$ ) and that the length $\Delta L$ of the hot zone was $40 \mu \mathrm{m}$.

The boundary conditions are as follows.

(1) Since the temperature distribution of the optical fiber is axisymmetric, the amount of heat conducted per unit area (heat flux) in the $r$ direction is set to 0 at the fiber center $(r=0)$ as follows:

$$
-\left.\lambda \frac{\partial T}{\partial r}\right|_{r=0}=0
$$

(2) At the outer fiber surface $\left(r=r_{f}\right)$, the amount of heat conducted per unit area (heat flux) is dissipated by radiative transfer or heat transfer to the open air and/or the jacketing layer $\left(T=T_{a}\right)$ as follows:

$$
\begin{aligned}
-\left.\lambda \frac{\partial T}{\partial r}\right|_{r=r_{f}}= & \sigma_{S} \epsilon_{e}\left(T^{4}-T_{a}^{4}\right) \\
& +\frac{\lambda}{\delta r_{t}}\left(T-T_{a}\right),
\end{aligned}
$$

where $\sigma_{S}$ is the Stefan-Boltzmann constant and $\epsilon_{e}(\sim 0.9)$ is the emissivity of the surface. $\delta r_{t}$ is the thickness of the thermal boundary layer and $\delta r_{t}=\delta r$ is assumed in our calculation, where $\delta r$ is the step size along the $r$-axis.

(3) At the center $(z=0)$ of the hot zone, the temperature of the fiber core center is $T_{c}^{0}$. Also, there is heat inflow along the $z$-axis only at the core center $(r=0)$, which is attributable to light absorption. Moreover, when $r \neq 0$ and $z=0$, there is neither heat inflow nor heat outflow along the $z$-axis. These conditions are given by

$$
-\left.\lambda \frac{\partial T}{\partial z}\right|_{z=0}= \begin{cases}\alpha I, & \text { if } r=0 \\ 0 & \text { if } r \neq 0\end{cases}
$$

(4) At both fiber ends $(z= \pm L)$, the amount of heat conducted per unit area (heat flux) is dissipated by radiation transfer to the open air $\left(T=T_{a}\right)$ as follows:

$$
-\left.\lambda \frac{\partial T}{\partial z}\right|_{z= \pm L}=\sigma_{S} \epsilon_{e}\left(T^{4}-T_{a}^{4}\right) .
$$

In contrast, as initial conditions, $T=T_{a}$ in the optical fiber at $t=0$, except in the hot zone, and the core-center temperature in the hot zone is equal to $T_{c}^{0}\left(>T_{a}\right)$ as follows:

$$
T(0, z, 0)= \begin{cases}T_{a}, & \text { if }-L \leq z<-\frac{\Delta L}{2}, \\ T_{c}^{0}, & \text { if }-\frac{\Delta L}{2} \leq z \leq \frac{\Delta L}{2}, \\ T_{a} & \text { if } \frac{\Delta L}{2}<z \leq L .\end{cases}
$$

When light propagates through the fiber core $(r=0)$ along the $z$ direction (away from the light source), the incident laser power $P$ decreases because of the nonzero optical absorption coefficient $\alpha$. When the laser light propagates from $z$ to $z+\delta z$ along the $z$-axis at $r=0$, the $P$ value is given by

$$
P=P_{0} \exp \left(-\alpha \delta z-\int_{-L}^{z} \alpha(T) d z\right)
$$

where $P_{0}$ is the initial laser power. The second term on the right-hand side expresses the optical absorption loss when the light propagates through a distance of $z-L$.

The results described above assume that the laser light propagates through the fiber core along the positive $z$ direction (away from the light source).

When the core layer is heated to above the vaporization point of silica $(\sim 3273 \mathrm{~K})$, an enclosed hollow cavity is produced in the core center. This cavity contains oxygen, which is produced by the pyrolysis reaction of (1). The heat conductivity $\kappa$ of the oxygen $\left(0.03 \mathrm{~W} \mathrm{~m}^{-1} \mathrm{~K}^{-1}\right)$ is two orders smaller than that of the silicate glass. Therefore, the heat transferred in the silica core is stopped at the cavity.

Moreover, as the cavity has a refractive index of $n \sim 1$, which is smaller than that of the silica core $\left(n_{1}=1.46\right)$, the light propagating in the core layer is reflected at the cavity wall. When the light direction is reversed at the cavity, the heat source term $\alpha I_{r}$ resulting from the optical absorption of the reflected light is added to $\alpha I$ in (13), where $I_{r}$ is the optical power intensity of the reflected laser light. 
We consider the $P$ value at a $z$ position located near the cavity wall. The length of this position is assumed to be $\delta z$. The laser light reaches $z$, propagates through a distance of $\delta z$, and then reaches the cavity wall, whose coordinate is $z_{v}$. Next, the light is reflected at the cavity wall and propagates in the negative $z$ direction and then reaches $z$ again. In such a case, the $P$ value at $z$ is given by

$$
\begin{aligned}
P= & P_{0} \exp \left(-\alpha \delta z-\int_{-L}^{z} \alpha(T) d z\right) \\
& \times R \exp \left(-2 \int_{z+\delta z}^{z_{v}} \alpha(T) d z\right),
\end{aligned}
$$

where $R$ is the reflectivity at the boundary of the silica core and the cavity. $R$ is given by

$$
R=\left(\frac{n_{1}-1}{n_{1}+1}\right)^{2}
$$

The second integral on the right-hand side of (26), which is related to the reflected light, slightly affects the occurrence of the fiber fuse. However, by taking this term into consideration, the calculated fiber fuse velocities fit the experimental values. For this reason, in the present work, we decided to take into account the effect of the reflected light.

In the following section, we describe the calculated time $(t)$ dependence of $T(r, z)$ in an SM optical fiber.

3.4. Propagation of Fiber Fuse in SM Optical Fiber. In the calculation, we used $T_{c}^{0}=2923 \mathrm{~K}$ and $T_{a}=298 \mathrm{~K}$. It was assumed that laser light of wavelength $\lambda_{0}=1.064 \mu \mathrm{m}$ and $P_{0}=$ $2 \mathrm{~W}$ was incident to an SMF-28 optical fiber, which has a core diameter of $8.2 \mu \mathrm{m}$, a refractive index difference $\Delta=0.36 \%$, and $A_{\text {eff }}=49.4091 \times 10^{-12} \mathrm{~m}^{2}$.

We calculated the $T(r, z)$ values at $t=1,11$, and $21 \mathrm{~ms}$ after the incidence of the $2 \mathrm{~W}$ laser light. The calculated results are shown in Figures 5-7, respectively.

As shown in Figure 5, the core center temperature near the end of the hot zone $(z \sim-0.7 \mathrm{~mm})$ changes abruptly to a large value of about $3.4 \times 10^{4} \mathrm{~K}$ after $1 \mathrm{~ms}$. This rapid rise in the temperature initiates the fiber fuse phenomenon as shown in Figures 6 and 7. After 11 and $21 \mathrm{~ms}$, the hightemperature front in the core layer reached $z$ values of -6.1 and $-11.5 \mathrm{~mm}$, respectively. The average propagation velocity $v_{f}$ was estimated to be $0.54 \mathrm{~m} / \mathrm{s}$ using these data.

When the laser light of $\lambda_{0}=1.064 \mu \mathrm{m}$ and $P_{0}=2 \mathrm{~W}$ is incident to the SMF-28 optical fiber, the optical power intensity is $I=4.048 \mathrm{MW} / \mathrm{cm}^{2}$. The fiber fuse velocity at this value of $I$ is estimated to be about $0.55 \mathrm{~m} / \mathrm{s}$ (see Figure 10 in [18]). This value is in very good agreement with the upper value obtained by calculation $(0.54 \mathrm{~m} / \mathrm{s})$.

On the other hand, Hand and Russell measured the fiber fuse temperature to be $5400 \mathrm{~K}$ [5], and Dianov et al. obtained a temperature of $4700-10500 \mathrm{~K}[23,56]$ by measurement. They estimated the temperatures from precisely measured spectral data in the $600-1400 \mathrm{~nm}[5]$ and $500-800 \mathrm{~nm}[23,56]$ regions, while assuming blackbody radiation.

In our calculation, the temperature distribution of the fiber fuse in the core center is shown in Figure 8. Similar



FIGURE 5: Temperature field in SMF-28 optical fiber after $1 \mathrm{~ms}$ when $P_{0}=2 \mathrm{~W}$ at $\lambda_{0}=1.064 \mu \mathrm{m}$.



FIgURE 6: Temperature field in SMF-28 optical fiber after $11 \mathrm{~ms}$ when $P_{0}=2 \mathrm{~W}$ at $\lambda_{0}=1.064 \mu \mathrm{m}$.

shapes of temperature distribution were reported by Kashyap et al. [13] and Facão et al. [33].

As shown in Figure 8, it is clear that the sharp temperature peak is located near the light source, and a relatively hightemperature plateau of about $5000 \mathrm{~K}$ extends over about $1.5 \mathrm{~mm}$ behind the sharp peak. This region, called the "radiation zone" in Figure 8, exhibits high temperatures of above $323 \mathrm{~K}$.

When gaseous $\mathrm{SiO}$ and/or $\mathrm{SiO}_{2}$ molecules are heated to high temperatures above $5000 \mathrm{~K}$, they decompose to form $\mathrm{Si}$ and $\mathrm{O}$ atoms and finally become $\mathrm{Si}^{+}$and $\mathrm{O}^{+}$ions and electrons in the ionized gas plasma state. The numbers of electrons and ions in the plasma front, which exhibits sharp temperature peak, are larger than those in the plateau region. However, as the plasma tends to restore electrical neutrality, the motions of the electrons and ions will not produce any change on the initial temperature distribution shown in Figure 8, except for the peak temperature reduction of the plasma front due to energy loss induced by electron-ion collisions.

In the ionized gas plasma, electron-ion collisions generate electromagnetic radiation because of the deceleration during the collisions. This bremsstrahlung emission $[57,58]$ is a universal and irreducible process of energy loss. If we assume that electrons mainly collide with ions with a charge of 




FIgURE 7: Temperature field in SMF-28 optical fiber after $21 \mathrm{~ms}$ when $P_{0}=2 \mathrm{~W}$ at $\lambda_{0}=1.064 \mu \mathrm{m}$.



Figure 8: Temperature distribution of the SMF-28 core center versus length along the $z$ direction when $P_{0}=2 \mathrm{~W}$ at $\lambda_{0}=1.064 \mu \mathrm{m}$. The time after the laser incidence is $10 \mathrm{~ms}$.

+1 in the plasma, the spectral radiance function $I_{p}$ for the bremsstrahlung emission is given by [59]

$$
I_{p}=\frac{N_{e}^{2} \nu^{2}}{\sqrt{T} c^{2}} \exp \left(-\frac{h v}{k T}\right)
$$

where $N_{e}$ is the number density of electrons in the plasma, $h$ is Planck's constant, and $v$ is the optical frequency. This function is directly proportionate to $\nu^{2}$ in the case of $h v \ll k T$.

On the other hand, in the case of $h v \ll k T$, the spectral radiance function $I_{b}$ for blackbody radiation is given by [60]

$$
I_{b}=\frac{2 \pi v^{2}}{c^{2}} k T \text {. }
$$

This is well known as the Rayleigh-Jeans formula, and this function is proportional to $v^{2}$, too. Therefore, we assumed that the radiation zone, in which the bremsstrahlung emission of the plasma is liberated, can be treated as a blackbody because of its similar dependence on $\nu$.

If we consider the radiation zone as a blackbody, that is, isolated from the surrounding nonheated regions, it is expected that the radiation zone will exhibit a radiation spectrum with a broad range of optical frequencies ranging



FIGURE 9: Average temperature of radiation zone versus time after fiber fuse generation.

from ultraviolet to infrared. The blackbody temerature $T_{b}$ of the zone is related to the frequency $v_{m}$ of the spectral peak as follows [60]:

$$
v_{m}=\frac{2.82 k T_{b}}{h} .
$$

If $T_{\mathrm{av}}$ is defined as the average temperature of the radiation zone, the $T_{b}$ will be close to the $T_{\mathrm{av}}$.

The relationship between the $T_{\mathrm{av}}$ value and the time after fiber fuse generation is shown in Figure 9. $T_{\text {av }}$ exceeds $10000 \mathrm{~K}$ immediately after fiber fuse generation but is less than $7000 \mathrm{~K}$ after $4 \mathrm{~ms}$. Then, it gradually approaches about $5700 \mathrm{~K}$. The value of $5760 \mathrm{~K}$ shown in Figure 9 is the average $T_{\mathrm{av}}$ value from 4 to $30 \mathrm{~ms}$. This temperature $(5760 \mathrm{~K})$ is close to the reported temperatures of $5400 \mathrm{~K} \mathrm{[5]} \mathrm{and} \mathrm{about} 5800 \mathrm{~K}$ [56].

Thus, it was found that if a fiber fuse, which exhibits a sharp temperature peak located near the light source, is approximated as a blackbody isolated from the surrounding nonheated regions, its average temperature from $4 \mathrm{~ms}$ after the generation of the fiber fuse approaches the experimentally estimated radiation temperatures.

\section{Conclusion}

We investigated the unsteady thermal conduction status in a single-mode optical fiber by numerical computation in order to visualize the mode of fiber fuse propagation. We assumed that the vitreous silica optical fiber underwent pyrolysis at elevated temperatures to form $\mathrm{SiO}_{x}(x \sim 1)$. We also proposed a model in which the optical absorption coefficient of the core layer increased with increasing molar concentration of $\mathrm{SiO}_{x}$. By using the model, we calculated the temperature distribution in the fiber with the explicit finitedifference method. It was found that when a short core with $40 \mu \mathrm{m}$ length was heated to $2923 \mathrm{~K}$ and a $2 \mathrm{~W}$ laser light (wavelength of $1.064 \mu \mathrm{m}$ ) entered the core layer of an SMF28 optical fiber, a thermal wave, that is, a fiber fuse, with 
a peak temperature of about $34000 \mathrm{~K}$ was generated at the boundary of the heating region near the light source. The fiber fuse was enlarged and propagated toward the light source at a rate of about $0.54 \mathrm{~m} / \mathrm{s}$. The calculated propagation velocity of the fiber fuse was in agreement with the experimental value. Moreover, the average temperature of the radiated region of the core layer was less than $7000 \mathrm{~K}$ at a time of $4 \mathrm{~ms}$ after the generation of the fiber fuse and gradually approached a temperature of about $5700 \mathrm{~K}$. The final average temperature was close to the experimentally reported values.

Recently, Toratani et al. demonstrated femtosecond laser fabrication of submicrometer-sized voids in fused silica, and both reflector and optical waveguides were fabricated by femtosecond laser only [61]. In this fabrication technique, self-focusing plays an important role to generate the voids. This technique would be useful to develop 3-dimensional optical devices and fabricate precise sensors. Our study may contribute somewhat to analyzing or controlling such a fabrication process.

The cause of mechanism of the fiber fuse phenomenon has yet to be sufficiently clarified. It largely depends upon future multilateral studies.

\section{Appendix}

\section{Focal Length of Thermal Lens Effect}

If part of the core layer is heated by light absorption, the refractive index gradient in the core is induced by the thermal coefficient of the refractive index $\partial n / \partial T$ of the silica glass and the temperature distribution $\Delta T$ in the core layer. The refractive index $n$ of the heated core is expressed as follows:

$$
n(r, t)=n_{0}+\frac{\partial n}{\partial T} \Delta T(r, t),
$$

where $n_{0}(=1.46)$ is the characteristic $n$ value of the core layer, $r$ is the radial distance from the center of the optical fiber, and $t$ is time, respectively.

If we assume that optical power in the optical fiber takes on Gaussian distribution, $\Delta T$ in the core layer is given by [35, 62]

$$
\Delta T(r, t)=\frac{\alpha P}{4 \pi \lambda}\left[\ln \left(1+\frac{8 D t}{\omega_{0}^{2}}\right)-\frac{16 D t}{\omega_{0}^{2}+8 D t} \frac{r^{2}}{\omega_{0}^{2}}\right],
$$

where $\alpha$ is the absorption coefficient, $P$ is the incident optical power, $\omega_{0}$ is the spot size radius of the laser beam, and $\lambda$ is the thermal conductivity of the silica glass, respectively. Parameter $D=\lambda / C_{p} \rho$, where $C_{p}$ and $\rho$ are the specific heat and density of the silica glass.

Substitution of (A.2) into (A.1) gives

$$
\begin{gathered}
n \cong n_{0}\left[1+\delta\left(\frac{r}{\omega_{0}}\right)^{2}\right], \\
\delta=-\frac{2 \alpha P}{4 n_{0} \pi \lambda}\left(\frac{\partial n}{\partial T}\right) \frac{8 D t}{\omega_{0}^{2}+8 D t} .
\end{gathered}
$$

When refractive index of the core layer takes a radial distribution as shown in (A.3), propagating laser beam will be focussed as a result of the thermal lens effect. In this case, the focal length $F$ is given by $[35,62]$

$$
\begin{gathered}
F(t)=\frac{n_{0} \pi \lambda \omega_{0}^{2}\left(\omega_{0}^{2}+8 D t\right)}{\alpha P l(\partial n / \partial T)(8 D t)}=F_{\infty}\left[1+\frac{t_{c}}{2 t}\right], \\
F_{\infty}=\frac{n_{0} \pi \lambda \omega_{0}^{2}}{\alpha P l(\partial n / \partial T)}, \\
t_{c}=\frac{\omega_{0}^{2}}{4 D},
\end{gathered}
$$

where $l$ is the length of the heating core, where $\alpha$ exhibits large value. In typical SM fiber, $\lambda=9.2 \mathrm{~W} / \mathrm{mK}, C_{p}=788 \mathrm{~J} / \mathrm{kgK}, \rho$ $=2200 \mathrm{~kg} / \mathrm{m}^{3}$ [5], and $\omega_{0} \sim 4.5 \mu \mathrm{m}$. If we insert these values into (A.5), we obtain $t_{c} \sim 0.95 \mu \mathrm{s}$. This means that $F \cong F_{\infty}$ when $t$ is $10 \mu$ s or above. If we assume $P=2 \mathrm{~W}, l \sim 40 \mu \mathrm{m}$, $\alpha=5.6 \times 10^{4} \mathrm{~m}^{-1}$ [5], and $\partial n / \partial T=1.23 \times 10^{-5} \mathrm{~K}^{-1}$ [5], $F$ is given by

$$
F \cong F_{\infty} \sim 15.5 \mu \mathrm{m}
$$

This $F$ value is of the same order of the observed interval and/or large front size of the cavities $[17,20]$.

\section{Conflict of Interests}

The author declares that there is no conflict of interests regarding the publication of this paper.

\section{References}

[1] A. Sano, T. Kobayashi, E. Yoshida, and Y. Miyamoto, "Ultra-high capacity optical transmission technologies for $100 \mathrm{Tbit} / \mathrm{s}$ optical transport networks," IEICE Transactions on Communications, vol. 94, no. 2, pp. 400-408, 2011.

[2] T. Morioka, "New generation optical infrastructure technologies: "EXAT initiative" towards 2020 and beyond," in Proceedings of the 14th OptoElectronics and Communications Conference (OECC '09), July 2009.

[3] R. Kashyap and K. J. Blow, "Observation of catastrophic selfpropelled self-focusing in optical fibres," Electronics Letters, vol. 24, no. 1, pp. 47-49, 1988.

[4] R. Kashyap, "Self-propelled self-focusing damage in optical fibres," in Proceedings of the 10th International Conference on Lasers, pp. 859-866, 1988.

[5] D. P. Hand and P. S. J. Russell, "Solitary thermal shock waves and optical damage in optical fibers: the fiber fuse," Optics Letters, vol. 13, no. 9, pp. 767-769, 1988.

[6] D. P. Hand and P. S. J. Russell, "Soliton-like thermal shockwaves in optical fibres: origin of periodic damage tracks," in Proceedings of the 14th European Conference on Optical Communication, pp. 111-114, September 1988.

[7] R. Kashyap, "The fiber fuse-from a curious effect to a critical issue: a 25th year retrospective," Optics Express, vol. 21, no. 5, pp. 6422-6441, 2013.

[8] S. Todoroki, "Fiber fuse propagation behavior," in Selected Topics on Optical Fiber Technology, Y. Moh, S. W. Harun, and H. Arof, Eds., pp. 551-570, InTech, Croatia, 2012. 
[9] P. André, A. Rocha, F. Domingues, and M. Facão, "Thermal effects in optical fibres," in Developments in Heat Transfer, M. A. D. Bernardes, Ed., pp. 1-20, InTech, Croatia, 2011.

[10] T. J. Driscoll, J. M. Calo, and N. M. Lawandy, "Explaining the optical fuse," Optics Letters, vol. 16, pp. 1046-1048, 1991.

[11] D. D. Davis Jr., S. C. Mettler, and D. J. DiGiovanni, "Experimental data on the fiber fuse," in 27th Annual Boulder Damage Symposium: Laser-Induced Damage in Optical Materials, vol. 2714 of Proceedings of SPIE, pp. 202-210, October 1995.

[12] D. D. Davis, S. C. Mettler, and D. J. DiGiovanni, "A comparative evaluation of fiber fuse models," in Laser-Induced Damage in Optical Materials, vol. 2966 of Proceedings of SPIE, pp. 592-606, October 1996.

[13] R. Kashyap, A. H. Sayles, and G. F. Cornwell, "Heat flow modeling and visualization of catastrophic self-propagating damage in single-mode optical fibers at low powers," in LaserInduced Damage in Optical Materials, vol. 2966 of Proceedings of SPIE, pp. 586-591, October 1996.

[14] E. M. Dianov, I. A. Bufetov, A. A. Frolov et al., "Catastrophic destruction of optical fibres of various composition caused by laser radiation," Quantum Electronics, vol. 32, no. 6, pp. 476478, 2002.

[15] E. M. Dianov, I. A. Bufetov, A. A. Frolov et al., "Catastrophic destruction of fluoride and chalcogenide optical fibres," Electronics Letters, vol. 38, no. 15, pp. 783-784, 2002.

[16] R. Kashyap, "High average power effects in optical fibres and devices," in Reliability of Optical Fiber Components, Devices, Systems, and Networks, vol. 4940 of Proceedings of SPIE, pp. 108117, October 2002.

[17] R. M. Atkins, P. G. Simpkins, and A. D. Yablon, "Track of a fiber fuse: a Rayleigh instability in optical waveguides," Optics Letters, vol. 28, no. 12, pp. 974-976, 2003.

[18] Y. Shuto, S. Yanagi, S. Asakawa, M. Kobayashi, and R. Nagase, "Fiber fuse phenomenon in step-index single-mode optical fibers," IEEE Journal of Quantum Electronics, vol. 40, no. 8, pp. 1113-1121, 2004.

[19] S.-I. Todoroki, "In-situ observation of fiber-fuse propagation," Japanese Journal of Applied Physics, vol. 44, no. 6, pp. 40224024, 2005.

[20] S.-I. Todoroki, "Origin of periodic void formation during fiber fuse," Optics Express, vol. 13, no. 17, pp. 6381-6389, 2005.

[21] E. D. Bumarin and S. I. Yakovlenko, "Temperature distribution in the bright spot of the optical discharge in an optical fiber," Laser Physics, vol. 16, no. 8, pp. 1235-1241, 2006.

[22] L. A. Bufetov, A. A. Frolov, E. M. Dianov, V. E. Fortov, and V. P. Efremov, "Dynamics of fiber fuse propagation," in Proceedings of the Optical Fiber Communication Conference (OFC/NFOEC '05), pp. 501-503, March 2005.

[23] E. M. Dianov, V. E. Fortov, I. A. Bufetov et al., "High-speed photography, spectra, and temperature of optical discharge in silica-based fiberss," IEEE Photonics Technology Letters, vol. 18, no. 6, pp. 752-754, 2006.

[24] S. Todoroki, "Fiber fuse propagation modes in typical singlemode fibers," in Proceedings of the Optical Fiber Communication Conference (OFC/NFOEC '13), 2013.

[25] S. I. Yakovlenko, "Plasma behind the front of a damage wave and the mechanism of laser-induced production of a chain of caverns in an optical fibre," Quantum Electronics, vol. 34, no. 8, pp. 765-770, 2004.

[26] A. M. Rocha, P. F. D. C. Antunes, M. D. F. F. Domingues, M. Facão, and P. S. D. B. André, "Detection of fiber fuse effect using
FBG sensors," IEEE Sensors Journal, vol. 11, no. 6, pp. 1390-1394, 2011.

[27] K. S. Abedin and M. Nakazawa, "Real time monitoring of a fiber fuse using an optical time-domain reflectometer," Optics Express, vol. 18, no. 20, pp. 21315-21321, 2010.

[28] D. P. Hand and T. A. Birks, "Single-mode tapers as "fibre fuse" damage circuit-breakers," Electronics Letters, vol. 25, no. 1, pp. 33-34, 1989.

[29] E. M. Dianov, I. A. Bufetov, and A. A. Frolov, "Destruction of silica fiber cladding by the fuse effect," Optics Letters, vol. 29, no. 16, pp. 1852-1854, 2004.

[30] S. Yanagi, S. Asakawa, M. Kobayashi, Y. Shuto, and R. Nagase, "Fiber fuse terminator," in Proceedings of the Pacific Rim Conference on Lasers and Electro-Optics, vol. 1, p. 386, 2003.

[31] K. Kurokawa and N. Hanzawa, "Suppression of fiber fuse propagation and its break in compact fiber fuse terminator," in Proceedings of the OECC/PS, pp. 4-5, 2013.

[32] S. I. Yakovlenko, "On reasons for strong absorption of light in an optical fibre at high temperature," Quantum Electronics, vol. 34, no. 9, pp. 787-789, 2004.

[33] M. Facão, A. M. Rocha, and P. S. De Brito André, “Traveling solutions of the fuse effect in optical fibers," Journal of Lightwave Technology, vol. 29, no. 1, pp. 109-114, 2011.

[34] E. M. Dianov, V. E. Fortov, I. A. Bufetov et al., "Detonationlike mode of the destruction of optical fibers under intense laser radiation," JETP Letters, vol. 83, no. 2, pp. 75-78, 2006.

[35] M. Katayama, Laser Chemistry; Nonlinear Spectroscopy and Laser Induced Chemical Processes, Syokabo, Tokyo, Japan, 1985.

[36] A. E. Owen and R. W. Douglas, "The electrical properties of vitreous silica," Journal of the Society of Glass Technology, vol. 43, pp. 159-178, 1959.

[37] R. H. Doremus, "Ionic transport in amorphous oxides," Journal of The Electrochemical Society, vol. 115, pp. 181-186, 1968.

[38] H. L. Schick, "A thermodynamic analysis of the hightemperature vaporization properties of silica," Chemical Reviews, vol. 60, no. 4, pp. 331-362, 1960.

[39] H. R. Philipp, "Optical properties of non-crystalline Si, SiO, $\mathrm{SiO}_{x}$ and $\mathrm{SiO}_{2}$," Journal of Physics and Chemistry of Solids, vol. 32, no. 8, pp. 1935-1945, 1971.

[40] H. R. Philipp, "Optical and bonding model for non-crystalline $\mathrm{SiO}_{x}$ and $\mathrm{SiO}_{x} \mathrm{~N}_{y}$ materials," Journal of Non-Crystalline Solids, vol. 8-10, pp. 627-632, 1972.

[41] W. J. Moore, Physical Chemistry, chapter 8, Prentice-Hall, 4th edition, 1972.

[42] JANAF Thermochemical Tables, U.S. Department of Commerce and National Bureau of Standards, 2nd edition, 1971.

[43] M. H. Brodsky, R. S. Title, K. Weiser, and G. D. Pettit, "Structural, optical, and electrical properties of amorphous silicon films," Physical Review B, vol. 1, no. 6, pp. 2632-2641, 1970.

[44] D. L. Dexter, "Interpretation of urbach's rule," Physical Review Letters, vol. 19, no. 24, pp. 1383-1385, 1967.

[45] H. Mahr, "Ultraviolet absorption of KI Diluted in KCl crystals," Physical Review, vol. 125, no. 5, pp. 1510-1516, 1962.

[46] G. G. Macfarlane and V. Roberts, "Infrared absorption of silicon near the lattice edge," Physical Review, vol. 98, no. 6, pp. 18651866, 1955.

[47] G. G. Macfarlane, T. P. McLean, J. E. Quarrington, and V. Roberts, "Fine structure in the absorption-edge spectrum of Si," Physical Review, vol. 111, no. 5, pp. 1245-1254, 1958. 
[48] E. Burstein, G. Picus, and N. Sclar, "Optical and photoconductive properties of silicon and germanium," in Proceedings of the Photoconductivity Conference, pp. 353-413, John Wiley \& Sons, New York, NY, USA, 1956.

[49] H. S. Carslaw and J. C. Jaeger, Conduction of Heat in Solids, chapter 13, Oxford University Press, Oxford, UK, 2nd edition, 1959.

[50] I. Barin and O. Knacke, Thermochemical Properties of Inorganic Substances, Springer, Berlin, Germany, 1973.

[51] M. Shoji, Heat Transfer Textbook, chapter 2, University Tokyo Press, Tokyo, Japan, 1995.

[52] T. Okuda, Plasma Engineering, chapter 3, Corona-Sha, Tokyo, Japan, 1975.

[53] T. Okuda, Plasma Engineering, chapter 1, Corona-Sha, Tokyo, Japan, 1975.

[54] L. Spitzer Jr., Physics of Fully Ionized Gases, John Wiley \& Sons, 2nd edition, 1962.

[55] G. D. Smith, Numerical Solution of Partial Differential Equations: Finite Difference Methods, chapter 2, Clarendon Press, Oxford, UK, 3rd edition, 1985.

[56] E. M. Dianov, V. E. Frotov, I. A. Bufetov et al., "Temperature of optical discharge under action of laser radiation in silica-based fibers," in Proceedings of the ECOC, 2005.

[57] L. Spitzer, Physics of Fully Ionized Gases, chapter 5, John Wiley \& Sons, 2nd edition, 1962.

[58] Y. B. Zel'dovich and Y. P. Raizer, Physics of Shock Waves and High-Temperature Hydrodynamic Phenomena, chapter 5, Dover, New York, NY, USA, 2002.

[59] T. Sekiguchi, Plasma Engineering, chapter 1, Ohm-Sha, Tokyo, Japan, 1997.

[60] G. R. Fowles, Introduction to Modern Optics, chapter 7, Dover, New York, NY, USA, 2nd edition, 1975.

[61] E. Toratani, M. Kamata, N. N. Nedyalkov, and M. Obara, "Femtosecond laser processing of subwavelength-sized voids for compact optical devices," in Commercial and Biomedical Applications of Ultrafast Lasers, vol. 6108 of Proceedings of SPIE, pp. 1-8, January 2006.

[62] J. P. Gordon, R. C. C. Leite, R. S. Moore, S. P. S. Porto, and J. R. Whinnery, "Long-transient effects in lasers with inserted liquid samples," Journal of Applied Physics, vol. 36, no. 1, pp. 3-8, 1965. 







The Scientific World Journal



Soft Matter


Submit your manuscripts at

http://www.hindawi.com



